\title{
ON THE GENERALIZED COMPLEMENTARITY PROBLEM
}

\author{
JEN-CHIH YAO'
}

(Received 3 April 1991; revised 26 July 1992)

\begin{abstract}
In this paper, the generalised complementarity problem studied by Parida and Sen [13] is further extended. The extended problem appears to be more general and unifying. Characterisations of solutions to this extended problem are given. Some existence results derived by these characterisations are presented. An application of the extended problem to the quasi-variational inequalities of obstacle type is considered.
\end{abstract}

\section{Introduction}

Let $f$ be a mapping of $\mathbf{R}^{n}$ into itself. The classical nonlinear complementarity problem is to find a vector $x \in \mathbf{R}^{n}$ such that $x \geq 0, f(x) \geq 0$ and $\langle x, f(x)\rangle=0$. The nonlinear complementarity problem was first introduced and studied in [3] (See also [4, 5]) where the notion of positively bounded Jacobians was introduced and the proof was constructed in the sense that an algorithm was employed to compute the unique solution. It is also worth noting that the nonlinear complementarity problem has many applications, for example, in control and optimisation, economics and transportation equilibrium, contact problems in elasticity, fluid flow through porous media, game theory, and mathematical programming.

In recent years, various extensions of the nonlinear complementarity problem have been proposed and analysed. See, for example, $[2,6,7,9,10,11,13$, 14]. In [13], Parida and Sen consider and study the following generalised complementarity problem:

${ }^{1}$ Dept of Appl. Mathematics, National Sun Yat-sen University, Kaohsiung, Taiwan 80424, R.O.C. (C) Australian Mathematical Society, 1994, Serial-fee code 0334-2700/94 
Find $x \in \mathbf{R}^{n}$ and $y \in F(x)$ such that

$$
x \in K, \quad \theta(x, y) \in K^{*} \quad \text { and } \quad\langle x, \theta(x, y)\rangle=0
$$

where $K$ is a closed convex cone in $\mathbf{R}^{n}, K^{*}=\left\{y \in \mathbf{R}^{n}:(y, x) \geq 0, \forall x \in K\right\}$ is the polar cone of $K, F$ is a point-to-set mapping from $K$ into $C \subset \mathbf{R}^{m}$ and $\theta$ is a point-to-point mapping from $K \times C$ into $\mathbf{R}^{n}$.

In this paper, we shall consider the following extension of the problem considered by Parida and Sen [13] by allowing $K$ to be a point-to-set mapping:

Find a vector $x \in m(x)+L(x)$ and a vector $y \in F(x)$ such that

$$
\theta(x, y) \in L(x)^{*} \text { and }\langle\theta(x, y), x-m(x)\rangle=0 \text {, }
$$

where $L$ is a cone-valued point-to-set mapping from a closed convex cone $K$ into itself and $m$ is a point-to-point mapping from $K$ into itself. We note that (2) reduces to the problem studied in [2] if $\theta(x, y)=y$ and $K=C=\mathbf{R}^{n}$. If we further assume that $L(x)=\mathbf{R}_{+}^{n}$ for all $x \in \mathbf{R}^{n}$ and $F$ is single-valued, then (2) reduces to the problem studied in [11]. If $m(x)=0$ and $L(x)=K$ for all $x \in K$, then (2) reduces to the problem studied in [13] and if we further assume that $C=\mathbf{R}^{n}$ and $\theta(x, y)=y$, then (2) reduces to the problem studied in [14]. Finally, if $F$ is further assumed to be single-valued, then (2) reduces to the problem studied in [9]. Therefore, it can be seen that (2) is a more general and more unifying problem.

We also note that a number of Kuhn-Tucker stationary point problems for nondifferentiable mathematical programming problems such as those studied in [13], [12] and [15] can be also cast into the form of (2).

In Section 2, we give some preliminaries that will be used throughout this paper. In Section 3, we first characterise solutions to (2). Some existence results are then derived from these characterisations. Finally, an application of (2) to the quasi-variational inequalities of obstacle type is considered.

\section{Preliminaries}

Let $X$ and $Y$ be metric spaces and $F$ be a point-to-set mapping from $X$ into $Y$. The mapping $F$ is said to be upper continuous at $x \in X[8]$ if and only if a sequence $\left\{x_{n}\right\}$ converging to $x$, and a sequence $\left\{y_{n}\right\}$ with $y_{n} \in F\left(x_{n}\right)$ converging to $y$, implies $y \in F(x)$. The mapping $F$ is said to be lower continuous at $x \in X$ [8] if and only if for any sequence $\left\{x_{n}\right\}$ converging to $x \in X$ and $y \in F(x)$, there 
exists an $n_{0}$ such that the sequence $\left\{y_{n}\right\}$ converges to $y \in Y$ and $y_{n} \in F\left(x_{n}\right)$ for all $n \geq n_{0}$. The mapping $F$ is said to be upper (lower) continuous if $F$ is upper (lower) continuous at every point $x \in X$ and $F$ is continuous if it is both upper and lower continuous. The point-to-set mapping $F$ is said to be uniformly compact near $x$ [8] if there exists a neighbourhood $V$ of $x$ such that $F(V)=\cup_{u \in V} F(u)$ is bounded. We say $F$ is uniformly compact on $X$ if it is uniformly compact near $x$ for all $x \in X$. For $K \subset \mathbf{R}^{n}, \operatorname{int}(K)$ and $\partial K$ denote the interior and boundary of $K$, respectively. For $K, B \subset \mathbf{R}^{n}$, int ${ }_{K}(B)$ and $\partial_{K}(B)$ denote the relative interior and relative boundary of $B$ in $K$, respectively.

REMARK. There are other notions of upper (lower) semicontinuity for point-toset mappings introduced by Berge [1] which are different from the notions of upper (lower) continuity defined above. But if the space $Y$ is compact and $F$ is upper continuous then $F$ is upper semicontinuous [1].

The following theorem will be needed in the next section.

THEOREM 2.1. Let $K \subset \mathbf{R}^{n}$ be nonempty compact convex and $C \subset \mathbf{R}^{m}$ be nonempty closed and convex. Let $X$ be a nonempty convex-valued continuous point-to-set mapping from $K$ into itself and $F$ be a convex-valued upper continuous and uniformly compact point-to-set mapping from $K$ into $C$. Let $\theta: K \times C \longrightarrow \mathbf{R}^{n}$ be continuous. Then there exist $x \in X(x)$ and $y \in F(x)$ such that $\langle\theta(x, y), u-x\rangle \geq 0$ for all $u \in X(x)$.

PROOF. Let $H$ be the closed convex hull of $\cup_{x \in K} F(x)$. Since $F$ is upper continuous and uniformly compact on $K$, it follows that $H$ is compact and convex. For each fixed $(x, y) \in K \times H$, the set

$$
V(x, y)=\left\{u \in X(x):\langle\theta(x, y), u-x\rangle=\min _{s \in X(x)}\langle\theta(x, y), s-x\rangle\right\}
$$

is convex. Let $G$ be a point-to-set mapping from $K \times H$ into itself defined by $G(x, y)=(V(x, y), F(x))$ for $(x, y) \in K \times H$. Then $G$ is upper continuous and $G(x, y)$ is compact and convex for all $(x, y) \in K \times H$. Now we can invoke the Kakutani Fixed Point Theorem to ensure that $G$ has a fixed point $(x, y)$ from which the result follows.

REMARK. Throughout the rest of this paper, it will be assumed that

1. $K$ is a closed convex cone in $\mathbf{R}^{n}$ and $C$ is a nonempty closed convex subset of $\mathbf{R}^{m}$, 
2. $m$ is a point-to-point mapping from $K$ into itself,

3. $L$ is a cone-valued point-to-set mapping from $K$ into itself,

4. $F$ is a point-to-set mapping from $K$ into $C$,

5. $X(x)=m(x)+L(x)$ for all $x \in K$,

6. For $r>0, X_{r}(x)=X(x) \cap B_{r}$ for all $x \in K$, where $B_{r}=\left\{x \in \mathbf{R}^{n}\right.$ : $\|x\| \leq r\}$,

7. $\theta$ is a continuous point-to-point mapping from $K \times C$ into $\mathbf{R}^{n}$.

\section{The main results}

First, we have the following simple but useful lemma.

LEMMA 3.1. Let $K$ be a nonempty convex subset of $\mathbf{R}^{n}$ and $D$ be any nonempty subset of $K$. If $x \in$ int $_{K}(D)$, then for any $u \in K$, there exists $a \lambda_{0}$ such that $0<\lambda_{0}<1$ and $\lambda x+(1-\lambda) u \in D$ for all $\lambda_{0} \leq \lambda \leq 1$.

PRoof. Since $x \in$ int $_{K}(D)$, there is an open set $U \subset \mathbf{R}^{n}$ so that $x \in K \cap U \subset$ int $_{K}(D)$. The result then follows from the facts that $\lambda x+(1-\lambda) u$ converges to $x$ as $\lambda$ converges to 1 and $U$ is an open neighbourhood of $x$.

We now have the following characterisations of a solution of (2).

THEOREM 3.2. The following statements are equivalent:

(i) a vector $x \in K$ is a solution to (2),

(ii) there exists an $r<0$ such that $x \in$ int $_{X(x)}\left(X_{r}(x)\right)$ and for some $y \in F(x)$

$$
\langle\theta(x, y), u-x\rangle \geq 0, \quad \forall u \in X_{r}(x),
$$

(iii) there exists an nonempty convex compact subset $B$ of $K$ such that $x \in$ int $_{X(x)}(X(x) \cap B$ ) and for some $y \in F(x)$

$$
\langle\theta(x, y), u-x\rangle \geq 0, \quad \forall u \in X(x) \cap B,
$$

(iv) $x \in X(x)$ and there is a $y \in F(x)$ such that

$$
\langle\theta(x, y), u-x\rangle \geq 0, \forall u \in X(x)
$$


Proof. (i) implies (ii). Suppose that $x \in K$ solves (2). Then $x \in X(x)$ and there exists $y \in F(x)$ such that

$$
\theta(x, y) \in L(x)^{*} \quad \text { and } \quad\langle\theta(x, y), x-m(x)\rangle=0 .
$$

Choose $r>0$ such that $x \in \operatorname{int}\left(B_{r}\right)$. Then $x \in X(x) \cap \operatorname{int}\left(B_{r}\right) \subset$ int $_{X(x)}\left(X_{r}(x)\right)$. For every $u \in X_{r}(x), u=m(x)+z$ for some $z \in L(x)^{*}$. Since $\theta(x, y) \in L(x)^{*}$, we have $\langle\theta(x, y), u-x\rangle=\langle\theta(x, y), z\rangle \geq 0$. Hence (ii) follows.

(ii) implies (iii). This is clear by letting $B=K \cap B_{r}$.

(iii) implies (iv). It follows immediately from the hypothesis that $x \in X(x)$. For any $u \in X(x)$, by Lemma 3.1, we can choose $\lambda>0$ so that $\lambda x+(1-\lambda) u \in$ $X_{r}(x)$. Then

$$
0 \leq\langle\theta(x, y), \lambda x+(1-\lambda) u-x\rangle=(1-\lambda)\langle\theta(x, y), u-x\rangle .
$$

Therefore $\langle\theta(x, y), u-x\rangle \geq 0$ for all $u \in X(x)$.

(iv) implies (i). Since $x-m(x) \in L(x)$ and $L(x)$ is a cone, $m(x), 2 x-m(x) \in$ $X(x)$. It then follows from (iv) that $\langle\theta(x, y), x-m(x)\rangle=0$. Finally, for any $z \in L(x)$, we have

$$
\langle\theta(x, y), z\rangle=\langle\theta(x, y), m(x)+z)-x\rangle \geq 0 .
$$

It follows that $\theta(x, y) \in L(x)^{*}$. Therefore $x$ is a solution to (2).

REMARK. If we consider the following generalised quasi-variational inequality problem: Find $x \in X(x)$ and $y \in F(x)$ such that

$$
\langle\theta(x, y), u-x\rangle \geq 0, \quad \forall u \in X(x),
$$

then the equivalence of (i) and (iv) in Theorem 3.2 states that both the solution sets of (3) and (2) are exactly the same. Such a nice relation between the variational inequality problem and the complementarity problem has already been investigated in [2], [9], [13] and [14].

Theorem 3.2 combined with Theorem 2.1 can be employed to obtain existence results for (2). To illustrate this assertion, we derive the following existence results for (2) by Theorem 3.2 and Theorem 2.1 .

THEOREM 3.3. Suppose that there exists an $r>0$ such that the following conditions hold:

(i) F is convex-valued, upper continuous and uniformly compact on $K \cap B_{r}$, 
(ii) $X_{r}$ is a nonempty convex-valued continuous point-to-set mapping on $K \cap B_{r}$,

(iii) for each $x \in X(x) \cap C_{r}$, there is a $u \in X(x) \cap$ int $_{K}\left(B_{r}\right)$ such that

$$
\max _{y \in F(x)}\langle\theta(x, y), u-x\rangle \geq 0 .
$$

Then there exists a solution to (2).

Proof. By Theorem 2.1, there exist $x \in X_{r}(x)$ and $y \in F(x)$ such that $\langle\theta(x, y), v-x\rangle \geq 0$ for all $v \in X_{r}(x)$. If $x \in$ int $_{X(x)}\left(X_{r}(x)\right)$, then the result follows from Theorem 3.1 (ii). Otherwise, $x \in X(x) \cap C_{r}$. By (iii), there exists a $u \in X(x) \cap \operatorname{int}_{K}\left(B_{r}\right)$ such that $\langle\theta(x, y), u-x\rangle \leq 0$ for all $y \in F(x)$. It follows that $\langle\theta(x, y), u-x\rangle=0$. For any $v \in X(x)$, choose $0<\lambda<1$ so that $\lambda u+(1-\lambda) v \in X_{r}(x)$. We then have

$$
0 \leq\langle\theta(x, y,), \lambda u+(1-\lambda) v-x\rangle=(1-\lambda)\langle\theta(x, y), v-x\rangle .
$$

Thus $\langle\theta(x, y), v-x\rangle \geq 0$. The result then follows from Theorem 3.2 (iv).

THEOREM 3.4. Suppose that there exists a nonempty compact subset $B$ of $K$ such that the following conditions hold:

(i) $F$ is convex-valued, upper continuous and uniformly compact on $B$,

(ii) $Y(x)=X(x) \cap B$ is a nonempty convex-valued continuous point-to-set mapping on $B$,

(iii) for each $x \in B$ and for each $x \in X(x) \backslash B$, there exists a vector $u \in Y(x)$ such that

$$
\max _{y \in F(x)}\langle\theta(x, y), u-z\rangle \leq 0 .
$$

Then there exists a solution to (2).

Proof. By Theorem 2.1, there exists $\bar{x} \in Y(\bar{x})$ and $\bar{y} \in F(\bar{x})$ such that $\langle\theta(\bar{x}, \bar{y}), x-\bar{x}\rangle \geq 0$ for all $x \in Y(\bar{x})$. Now for $x \in X(\bar{x}) \backslash B$, by condition (iii), there exists a $u \in Y(\bar{x})$ such that $\langle\theta(\bar{x}, \bar{y}), x-u\rangle \geq 0$. On the other hand, we have $\langle\theta(\bar{x}, \bar{y}), u-\bar{x}\rangle \geq 0$. By adding the last two inequalities, we have $\langle\theta(\bar{x}, \bar{y}), x-\bar{x}\rangle \geq 0$ The result then again follows from Theorem 3.2 (iv).

The following corollary follows immediately from Theorem 3.4 by letting $C=K, X$ be a constant point-to-set mapping with $X(x)=K$ for all $x \in K$ and $\theta(x, y)=y$. 
COROLLARY 3.5. Suppose that there exists a nonempty compact subset $B$ of $K$ such that the following conditions hold:

(i) $F$ is convex-valued upper continuous and uniformly compact on $B$,

(ii) for each $z \in K \backslash B$, there exists a vector $u \in B$ such that

$$
\max _{y \in F(x)}\langle y, u-z\rangle \leq 0 \text {. }
$$

Then there exist $x \in K$ and $Y \in F(x)$ such that $y \in K^{*}$ and $\langle y, x\rangle=0$.

Corollary 3.5 extends [14, Theorem 2.1] where strict inequality in (ii) is required. Finally, we have

THEOREM 3.6. Suppose that

(i) $F$ is convex-valued upper continuous and uniformly compact on $K$,

(ii) there exists a vector $x_{0} \in \cap_{x \in K} X(x)$ such that

$$
\liminf _{\|x\| \rightarrow \infty, x \in X(x)} \max _{y \in F(x)}\left\langle\theta(x, y), x_{0}-x\right\rangle<0,
$$

(iii) there exists an $r_{0}>0$ such that $X_{r}$ is a nonempty, convex-valued continuous point-to-set mapping for all $r \geq r_{0}$.

Then there exists a solution to (2).

PROOF. By (ii), there exists an $r_{1}>0$ such that for any $x \in X(x)$ and $\|x\| \geq r_{1}$, we have

$$
\max _{y \in F(x)}\left\langle\theta(x, y), x_{0}-x\right\rangle<0 .
$$

Let $r \geq \max \left\{r_{0}, r_{1},\left\|x_{0}\right\|\right\}$. Then by Theorem 2.1, there exist $x \in X_{r}(x)$ and $y \in F(x)$ such that $\langle\theta(x, y), u-x\rangle \geq 0$ for all $u \in X_{r}(x)$. It follows from (4) that $x \in X(x) \cap$ int $\left(B_{r}\right) \subset$ int $_{X(x)}\left(X_{r}(x)\right)$. Consequently, the result follows from Theorem 3.2 (ii).

In the case where the point-to-set mapping $L$ is constant with $L(x)=L_{0}$ for all $x \in K$, the condition that $X_{r}$ is continuous on $K \cap B_{r}$ for large $r$ is automatically true if the function $m$ is continuous. We have

THEOREM 3.7. Let $L_{0}$ be a closed solid cone in $\mathbf{R}^{n}$. Suppose that

(i) $F$ is convex-valued upper continuous and uniformly compact on $K$,

(ii) the function $m$ is continuous on $K$,

(iii) there exists a vector $u_{0} \in K$ such that $u_{0}-m(x) \in L_{0}, \forall x \in K$,

(iv) $\liminf \|x\| \rightarrow \infty, x \in X(x) \max _{y \in F(x)}\left\langle\theta(x, y), u_{0}-x\right\rangle<0$.

Then there exists a solution to (2). 
PROOF. Condition (iii) implies that $u_{0} \in \cap_{x \in K} X(x)$. The fact that $X_{r}$ is continuous for sufficiently large $r$ can be proved by a standard argument as that in [2, Theorem 4.2]. Hence the result follows from Theorem 3.6.

Before closing this section, we give an application of (2) to the quasivariational inequalities of obstacle type.

Let $K$ be a closed convex cone in $\mathbf{R}^{n}$ and $\leq_{K}$ be the partial order induced by $K$, that is, $x \leq_{K} y$ if and only if $x-y \in K$ for all $x, y \in \mathbf{R}^{n}$. Let $f, m$ be functions from $\mathbf{R}^{n}$ into itself. The quasi-variational inequality problem of obstacle type is to find $x^{*} \in \mathbf{R}^{n}$ such that

$$
x^{*} \geq_{K} m\left(x^{*}\right), \quad\left\langle f(x), x-x^{*}\right\rangle \geq 0, \quad \forall x \leq_{K} m\left(x^{*}\right) .
$$

It is interesting to note that if $K=\mathbf{R}_{+}^{n}$ and $m(x)=0$ for all $x \in \mathbf{R}^{n}$, then problem (5) is equivalent to a nonlinear complementarity problem. We now associate with problem (5) the following complementarity problem of form (2). Let $X$ be a point-to-set mapping from $\mathbf{R}^{n}$ into itself defined as $X(x)=m(x)+K$ for all $x \in \mathbf{R}^{n}$. Find $x^{*} \in m\left(x^{*}\right)+K$ such that

$$
f\left(x^{*}\right) \in K^{*}, \quad\left\langle f\left(x^{*}\right), x^{*}-m\left(x^{*}\right)\right\rangle=0 .
$$

It is easy to see that problem (5) is equivalent to problem (6) by Theorem 3.2. Consequently, we have the following existence result for problem (5).

THEOREM 3.8. Let $K$ be a closed solid convex cone in $\mathbf{R}^{n}$. Let $f$ and $m$ be continuous functions from $\mathbf{R}^{n}$ into itself and $X(x)=m(x)+K$ be a point-to-set mapping from $\mathbf{R}^{n}$ into itself. Suppose that there exists a vector $u_{0} \in \mathbf{R}^{n}$ such that $u_{0}-m(x) \in K, \forall x \in \mathbf{R}^{n}$ and

$$
\liminf _{\|x\| \rightarrow \infty, x \in X(x)}\left\langle f(x), x_{0}-x\right\rangle<0 .
$$

Then there exists a solution to problem (5).

PROOF. This result follows from Theorem 3.7 and the note above.

We conclude this section by noting that one possible generalisation of the above results is that the convex-valued assumption of the point-to-set mapping $F$ can be weakened to be contractible-valued. 


\section{References}

[1] C. Berge, Topological Spaces (Oliver \& Boyd, Edinburgh/London, 1963).

[2] D. Chan and J. S. Pang, "The generalized quasi-variational inequality problem", Math. Oper. Res. 7 (1982) 211-222.

[3] R. W, Cottle, "Nonlinear programs with positively bounded Jacobians", SIAM J. Appl. Math. 14 (1966) 147-158.

[4] R. W. Cottle, "Complementary and variational problems", Sympos. Math. 19 (1976) $177-$ 208.

[5] R. W. Cottle and G. B. Dantzig, "Complementary pivot theory of mathematical programming”, Linear Algebra Appl. 1 (1968) 103-125.

[6] G. J. Habetler and A. J. Price, "Existence theory for generalized nonlinear complementarity problems", J. Optim. Theory Appl. 7 (1971) 229-239.

[7] P. T. Harker and J. S. Pang, "Finite-dimensional variational inequality and nonlinear complementarity problems: A survey of theory, algorithms and applications", Math.Programming 48 (1990) 161-220.

[8] W. W. Hogan, "Point-to-set maps in mathematical programming", SIAM Rev. 15 (1973) 591-603.

[9] S. Karamardian, "Generalized complementarity problems", J. Optim. Theory Appl.3(1971) 161-168.

[10] S. Karamardian, "Complementarity problems over cones with monotone and pseudomonotone maps", J. Optim. Theory Appl. 18 (1976) 445-454.

[11] M. A. Noor and S. Zarae, "Linear quasi complementarity problems", Utilitas Math. 27 (1985) 249-260.

[12] J. Parida and A. Sen, "Duality and existence theory for nondifferentiable programming", J. Optim. Theory Appl. 48 (1986) 451-458.

[13] J. Parida and A. Sen, "A variational-like inequality for multifunctions with applications", J. Math. Anal. Appl. 124 (1987) 73-81.

[14] R. Saigal, "Extension of the generalized complementarity problem", Math. Oper. Res. 1 (1976) 260-266.

[15] M. Schechter, "A subgradient duality theorem", J. Math. Anal. Appl. 61 (1977) 850-855. 